\title{
Testing for a break in persistence under long-range dependencies and mean shifts*
}

\author{
Philipp Sibbertsen ${ }^{\dagger}$ and Juliane Willert \\ Institute of Statistics, Faculty of Economics and Management \\ Leibniz Universität Hannover, 30167 Hannover, Germany
}

\begin{abstract}
We show that the CUSUM-squared based test for a change in persistence by Leybourne et al. (2007) is not robust against shifts in the mean. A mean shift leads to serious size distortions. Therefore, adjusted critical values are needed when it is known that the data generating process has a mean shift. These are given for the case of one mean break. Response curves for the critical values are derived and a Monte Carlo study showing the size and power properties under this general de-trending is given.
\end{abstract}

Keywords: Break in persistence, long memory, structural break, level shift.

JEL-Codes: C12, C22

\section{Introduction}

It is well known that structural breaks in the mean of a time series can easily be confused with long-range dependence. Shifts in the mean can heavily bias estimators for the memory parameter and therefore create misleading results. For an overview about the problem of spurious long memory due to mean shifts see Sibbertsen (2004). In the recent years a change of the persistence of a time series, this is a change of the order of integration, has come more

${ }^{*}$ We would like to thank Robinson Kruse and the participants of the Pfingsttagung 2009 of the German Statistical Society in Merseburg for their helpful comments and suggestions.

${ }^{\dagger}$ Corresponding author, sibbertsen@statistik.uni-hannover.de 
and more into the focus of empirical and theoretical researchers. Beginning with Banerjee et al. (1992) several authors proposed tests for a change in persistence in the classical $I(0) / I(1)$ framework. A popular stationarity test against a break in persistence was introduced by Kim (2000). Kim's test has the disadvantage to reject the null if the data generating process is constantly $I(1)$ during the whole sample what is theoretically correct but not desirable. Leybourne et al. (2007) suggest a CUSUM-squares based test to solve this problem. Sibbertsen and Kruse (2009) generalized this test to the long memory framework by allowing for fractional degrees of integration.

Belaire-Franch (2005) proved that Kim's test is not robust against mean shifts in the sense that it has an asymptotic size of one when the data generating process is $I(0)$ with a break in the mean. Unfortunately, we show that the Leybourne et al. test does not overcome this problem as it is not robust against mean shifts either. We therefore derive adjusted critical values for the test under a generalized de-trending allowing for one mean shift.

The rest of the paper is organized as follows. In section 2 the test for changes in persistence is briefly described. Section 3 derives its properties under mean shifts and section 4 contains some Monte Carlo studies. Section 5 gives critical values of the test under a generalized de-trending procedure. Size and power results are given as well. Section 6 concludes.

\section{Testing for a break in persistence under long memory}

We assume that the data generating process follows an $\operatorname{ARFIMA}(0, d, 0)$ process. Sibbertsen and Kruse (2009) generalized a CUSUM of squares-based type test proposed by Leybourne et al. (2007) to test in this model framework the hypothesis of constant long-range dependencies versus a change in persistence. The alternative can be either a change in persistence from stationary to non-stationary long memory or vice versa. The null hypothesis tested is

$$
H_{0}: d=d_{0} \quad \text { for } \quad t=1, \ldots, T,
$$


where we assume $1 / 2<d_{0}<3 / 2$. The alternative hypothesis is either

$$
H_{01}: \begin{cases}d=d_{1} \in(0,1 / 2) & \text { for } t=1, \ldots,[\tau T] \\ d=d_{2} \in(1 / 2,3 / 2) & \text { for } t=[\tau T]+1, \ldots, T\end{cases}
$$

or

$$
H_{10}: \begin{cases}d=d_{2} \in(1 / 2,3 / 2) & \text { for } t=1, \ldots,[\tau T] \\ d=d_{1} \in(0,1 / 2) & \text { for } t=[\tau T]+1, \ldots, T .\end{cases}
$$

The CUSUM of squares-based test statistic $R$ used in Sibbertsen and Kruse (2009) is given by

$$
R=\frac{\inf _{\tau \in \Lambda} K^{f}(\tau)}{\inf _{\tau \in \Lambda} K^{r}(\tau)}
$$

with the forward statistic

$$
K^{f}(\tau)=[\tau T]^{-2 d_{0}} \sum_{t=1}^{[\tau T]} \hat{v}_{t, \tau}^{2}
$$

and the reversed statistic of the data generating process

$$
K^{r}(\tau)=(T-[\tau T])^{-2 d_{0}} \sum_{t=1}^{T-[\tau T]} \tilde{v}_{t, \tau}^{2} .
$$

Here $\tau$ is the relative breakpoint where we assume that $\tau \subset \Lambda$ and $\Lambda \subset(0,1)$ and is symmetric around 0.5 . For now we assume $\tau$ to be fixed though unknown. $[x]$ is the ceiling function of $\mathrm{x}$ and $\hat{\mathrm{v}}_{t, \tau}$ is the residual from the OLS regression of $X_{t}$ on a constant $z_{t}=1 \forall t$ based on the observations up to $[\tau T]$. This is

$$
\hat{v}_{t, \tau}=X_{t}-\bar{X}(\tau)
$$

with $\bar{X}(\tau)=[\tau T]^{-1} \sum_{t=1}^{[\tau T]} X_{t}$. Similarly $\tilde{v}_{t, \tau}$ is defined for the reversed series $y_{t}=X_{T-t+1}$. Thus, it is given by

$$
\tilde{v}_{t, \tau}=y_{t}-\bar{y}(1-\tau)
$$

with $\bar{y}(1-\tau)=(T-[\tau T])^{-1} \sum_{t=1}^{T-[\tau T]} y_{t}$.

Sibbertsen and Kruse (2009) derive the limiting distribution of this test statistic and provide response curves in order to compute critical values for different hypothetical memory parameters $d_{0}$. 


\section{Behavior of Test under mean shifts}

In order to analyze how the CUSUM of squares-based test behaves under mean shifts let us introduce some notation first. In what follows $\tau$ denotes the relative breakpoint in the memory parameter $d$ and $\lambda$ denotes the relative position of the mean shift. For the sake of notational simplicity we only consider the easiest break in mean model allowing only for abrupt changes. Our model is given by

$$
y_{t}=\alpha+\delta D_{t}+\varepsilon_{t}
$$

with $D_{t}=1(t \geq[\lambda T]+1)$ with $1(\cdot)$ being the indicator function. In this model a level shift from $\alpha$ to $\delta$ occurs at some unknown breakpoint $[\lambda T]$. We further assume that $\varepsilon_{t} \sim I(d)$ with $0 \leq d \leq 1.5$. Thus, a possible choice for $\varepsilon_{t}$ is an $\operatorname{ARFIMA}(p, d, q)$ model. Let furthermore $\stackrel{P}{\rightarrow}$ denote convergence in probability.

Theorem 1. Given model (1) with the assumptions given above. Then:

1. for $1 / 2<d<3 / 2$ the value of the test statistic is

$$
R=\frac{\inf _{\tau \in \Lambda} K^{f}(\tau)}{\inf _{\tau \in \Lambda} K^{r}(\tau)}=\frac{\inf _{\tau \leq \lambda} K^{f}(\tau)}{\inf _{\tau \geq \lambda} K^{r}(\tau)}
$$

2. for $0 \leq d<1 / 2$ we have $R \stackrel{P}{\rightarrow} 1$.

The results can also be derived for a general de-trending. The ideas are the same. It only introduces more notational difficulties and is therefore left out here.

The result means that the minimization takes place over a restricted interval up to the point where the mean shift occurs or beginning from this point. The further the mean shift is on the limits of $\Lambda$ the smaller is this interval either for the forward or reversed statistic. Therefore, the occurrence of the minimum in this interval becomes less likely. This can be seen when considering a typical shape of the forward and reversed statistic as given in Figure 1 . At $\lambda=$ 0.7 the forward statistic increases immediately and so the minimum can only be found before the mean shift distorts the forward statistic. This distortion is big enough for the test statistic to reject the null in most cases. It should be mentioned that we cannot prove inconsistency of the test in the sense that the test statistic diverges when a mean shift occurs. This is not the 
case and thus allows us to readjust the critical values in the case of mean shifts as it is done in section 5 .

Figure 1: Forward and backward statistic with $\lambda=0.7, \alpha=0, \delta=5$ and $d=0.8$
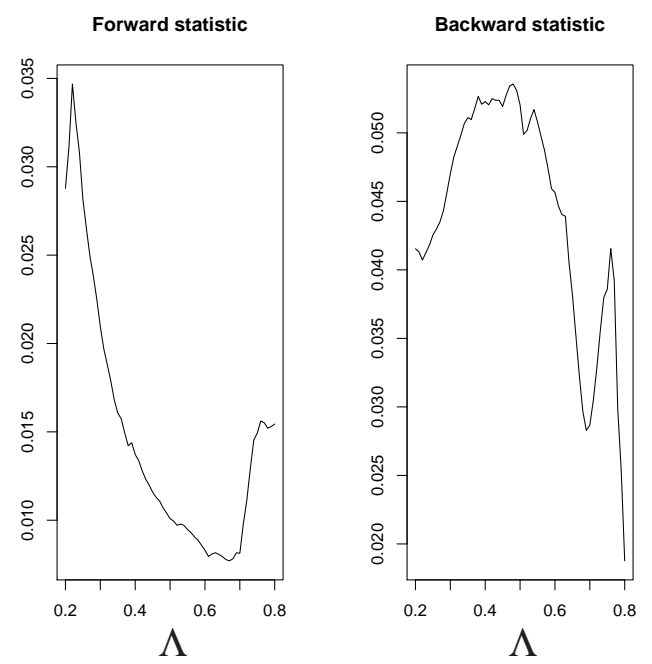

The size distortions are smallest for a mean shift at $\lambda=0.5$ considering that the interval for the forward and backward statistic have the same length. Therefore, it is less likely that both minima findings are distorted. Interestingly, these results do not hold for a stationary data generating process. In this case the test statistic is still conservative.

Some Monte Carlo underpinning these findings is given in the next section.

\section{Monte Carlo study}

Our theoretical findings in section 3 can be backed up with Monte Carlo studies. All simulations are computed with the open-source programming language R (2008). The number of replications is set to $M=2000$ and we consider a sample size of $T=1000$, set so high in order to illustrate the asymptotic results. When there is a mean shift from $\alpha=0$ to $\delta=5$ in model (1), the size varies with the relative position of the mean shift $\lambda$ as follows.

As shown in section 3 it leads to distorted size results for $1 / 2<d<3 / 2$ no matter what shift size is used. For $d<1$ it remains most likely above the significance level. The size distortion increases by getting closer to the limits of the $\Lambda$ interval. For $d=1$ as well as for $\lambda=0.5$ 
Table 1: Empirical size when there is a mean shift using estimated response curves

\begin{tabular}{r|rrrrr|rrrrr}
\hline \hline & \multicolumn{5}{|c|}{$d=0.6$} & \multicolumn{5}{c}{$d=0.8$} \\
\hline$\lambda$ & 0.10 & 0.25 & 0.50 & 0.75 & 0.90 & 0.10 & 0.25 & 0.50 & 0.75 & 0.90 \\
$1 \mathrm{~L}$ & 0.15 & 0.00 & 3.75 & 43.95 & 34.30 & 0.35 & 0.60 & 1.00 & 2.60 & 2.90 \\
$5 \mathrm{~L}$ & 0.70 & 0.65 & 11.75 & 67.45 & 57.20 & 2.40 & 3.05 & 5.15 & 10.00 & 11.60 \\
$10 \mathrm{~L}$ & 1.20 & 1.05 & 19.45 & 77.15 & 71.35 & 5.35 & 7.00 & 10.55 & 17.95 & 19.90 \\
$10 \mathrm{U}$ & 70.40 & 74.80 & 15.80 & 0.50 & 0.75 & 19.20 & 18.45 & 10.05 & 6.75 & 6.60 \\
$5 \mathrm{U}$ & 55.25 & 62.45 & 8.40 & 0.10 & 0.4 & 12.00 & 11.70 & 5.60 & 3.30 & 3.05 \\
$1 \mathrm{U}$ & 30.45 & 38.30 & 2.65 & 0.00 & 0.10 & 3.40 & 2.70 & 0.80 & 0.60 & 0.50 \\
\hline & & & $d=1.0$ & & & & & $d=1.4$ & & \\
\hline$\lambda$ & 0.10 & 0.25 & 0.50 & 0.75 & 0.90 & 0.10 & 0.25 & 0.50 & 0.75 & 0.90 \\
$1 \mathrm{~L}$ & 0.75 & 1.55 & 1.20 & 1.20 & 0.85 & 0.15 & 0.20 & 0.15 & 0.1 & 0.15 \\
$5 \mathrm{~L}$ & 4.10 & 6.00 & 5.10 & 4.90 & 4.90 & 2.25 & 2.70 & 2.15 & 1.70 & 2.00 \\
$10 \mathrm{~L}$ & 9.30 & 11.95 & 9.60 & 10.05 & 10.45 & 6.55 & 5.65 & 5.50 & 4.3 & 5.25 \\
$10 \mathrm{U}$ & 9.45 & 8.65 & 9.65 & 10.35 & 10.40 & 5.55 & 7.00 & 6.55 & 6.45 & 6.1 \\
$5 \mathrm{U}$ & 4.60 & 4.85 & 5.40 & 5.00 & 6.30 & 2.00 & 2.55 & 2.45 & 2.55 & 2.4 \\
$1 \mathrm{U}$ & 1.00 & 1.30 & 1.20 & 1.00 & 1.00 & 0.00 & 0.30 & 0.10 & 0.15 & 0.05 \\
\hline \hline
\end{tabular}

the smallest size distortion can be observed. For $d>1$ the test statistic tends to conservative size results. The test statistic does not diverge because of a mean shift and tends to reject not properly. Because of the missing mean reverting characteristic for long memory with $d>1$ and the thereby explosive performance of the time series, the mean shift no matter what size has no such strong impact on the test statistic and hence on the size results.

For this onesided test depending on whether $\lambda$ is smaller or greater than the interval $\Lambda$, elevated size values appear at the upper and lower bound respectively as shown in Figures 2 and 3. Due to the fact, that the true position of the break is unknown, distorted size results can always appear. 
Figure 2: Behavior of the size at the lower $5 \%$ tail

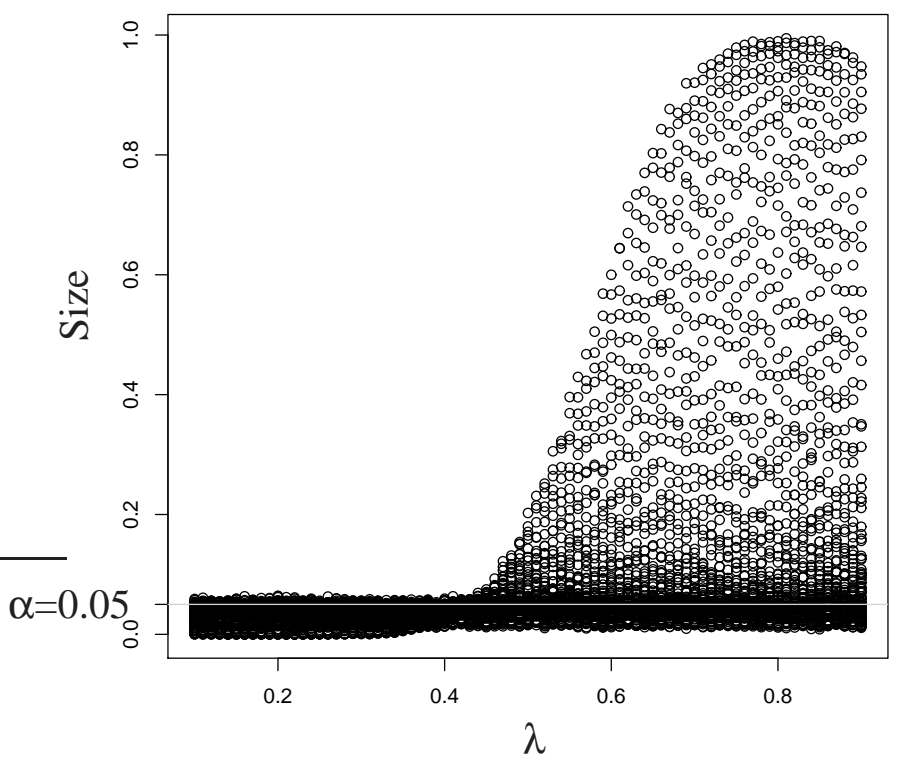

Figure 3: Behavior of the size at the upper $5 \%$ tail

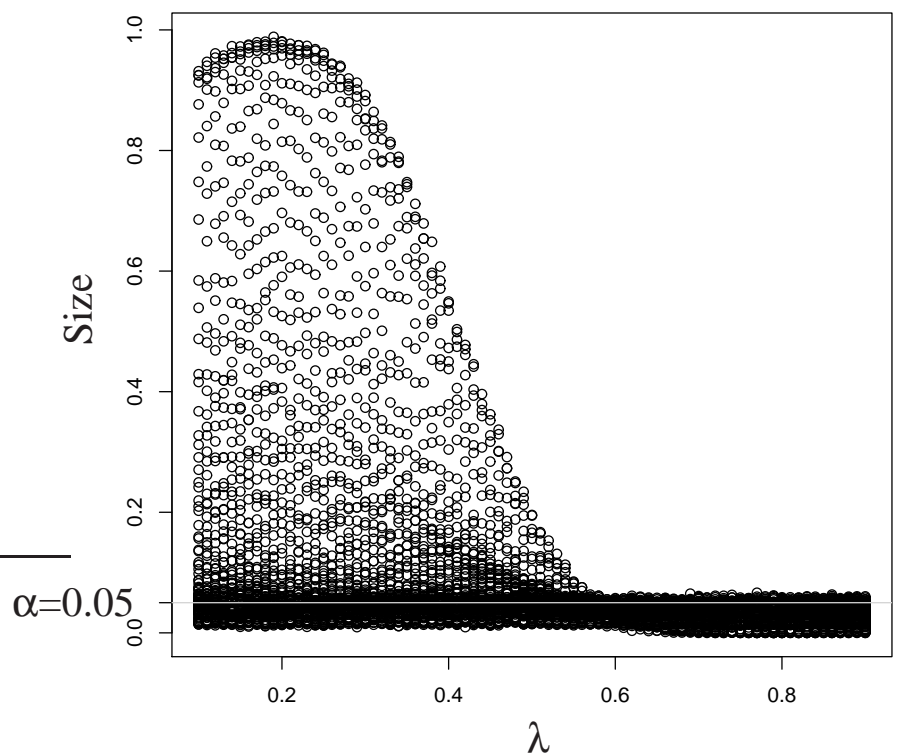

The smaller the long memory parameter, the more distinctive is this size behavior. Hence at the boundaries of the time series the test decision is strongly biased by the mean shift and leads to a false rejection of the null. The following graphic show the distribution of the minima of the forward and backward statistics for $\lambda=0.7$ and $d=0.8$. It shows that the minima of the forward statistic cumulate at the boundary of 0.8 and around $\lambda=0.7$. The reversed statistic shows similar findings with a cumulation at 0.2 . 
Figure 4: Empirical minima of the forward and backward statistics
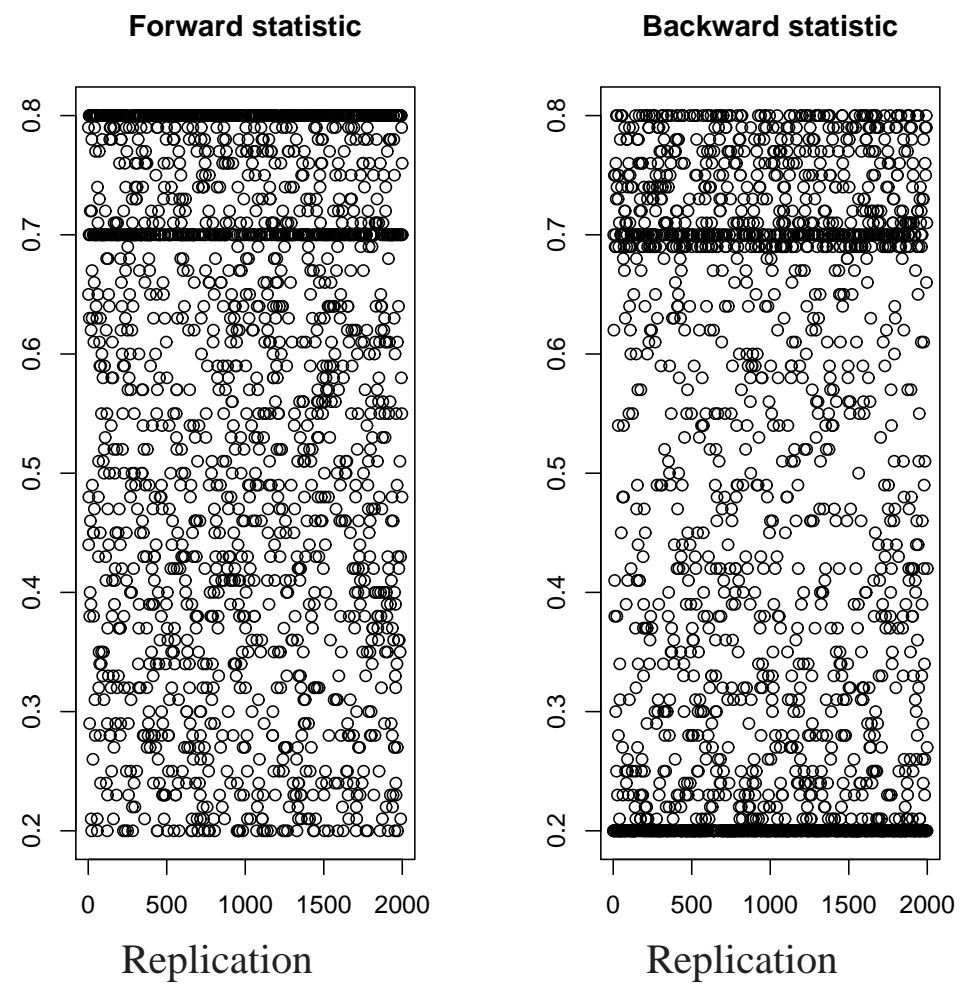

\section{Adjustment of critical values}

Due to the size distortion at the boundaries it is reasonable to adjust the critical values and take the mean shifts into account. The adjustment of the critical values takes place under the allowance for one break in the mean. It should be mentioned that for our adjustment procedure the existence of the mean shift has to be known. Estimating mean shifts within a long memory model with breaking persistence is a difficult task and beyond the scope of this paper. It should be mentioned that the response curves given in this chapter and thus the critical values of the test depend on $\lambda$. However, as in most applications there are at least rough if not exact ideas about mean shifts in the data, we consider our procedure still as useful for the practitioner.

We simulate the asymptotic distribution of the test statistic depending on $d$ for the cases $d=$ 0.51 to $d=1.49$ with $\lambda=0.5$. Due to the wide range of possible values of $d$ we fit polynomial 
functions to the sequence of critical values depending on $d$. The adjusted critical values can be displayed in response curves given by

$$
q_{\alpha}(d)=\sum_{i=0}^{s} \beta_{i} d^{i} .
$$

$q_{\alpha}$ denotes the $\alpha$-quantile of the asymptotic distribution and s the maximal polynomial order which is set to nine. The parameters $\beta_{i}$ are estimated with OLS. For different values of $\lambda$ the response curves are parallel so the functional form remains unchanged for different values of $\lambda$ though the parameters change.

Table 2: Estimated response curve when a mean shift occurs

\begin{tabular}{|c|c|c|c|c|c|c|c|c|c|c|}
\hline & $\beta_{0}$ & $\beta_{1}$ & $\beta_{2}$ & $\beta_{3}$ & $\beta_{4}$ & $\beta_{5}$ & $\beta_{6}$ & $\beta_{7}$ & $\beta_{8}$ & $\beta_{9}$ \\
\hline $1 \mathrm{~L}$ & 0.965 & 0 & 0 & 0 & -21.507 & 66.386 & -89.993 & 65.334 & -24.904 & 3.927 \\
\hline $5 \mathrm{~L}$ & -162.657 & 1700.856 & -7729.333 & 20149.046 & -33212.416 & 35896.289 & -25444.859 & 11411.826 & -2940.170 & 331.773 \\
\hline $10 \mathrm{~L}$ & 0.931 & 0 & 0 & 0 & -2.550 & 2.475 & 0 & 0 & -0.675 & 0.283 \\
\hline $10 \mathrm{U}$ & 1.132 & 0 & 0 & 0 & 0 & 0 & 10.268 & -18.031 & 11.346 & -2.557 \\
\hline $5 \mathrm{U}$ & 1.161 & 0 & 0 & 0 & 0 & 0 & 16.821 & -30.738 & 20.506 & -4.932 \\
\hline $1 \mathrm{U}$ & 0.975 & 0 & 0 & 0 & 18.784 & -41.418 & 39.564 & -13.136 & 0 & 0 \\
\hline
\end{tabular}

The size and power properties of the test using the estimated response curves for one break in the mean are reported in Tables 3 and 4, respectively.

Table 3: Empirical size

\begin{tabular}{r|lclr}
\hline \hline $\mathrm{d}$ & 0.55 & 0.70 & 0.85 & 1.25 \\
\hline $1 \mathrm{~L}$ & 0.4 & 1.0 & 0.7 & 2.2 \\
$5 \mathrm{~L}$ & 3.3 & 5.3 & 3.9 & 3.4 \\
$10 \mathrm{~L}$ & 8.5 & 9.7 & 9.8 & 8.1 \\
$10 \mathrm{U}$ & 10.4 & 9.3 & 11.1 & 10.2 \\
$5 \mathrm{U}$ & 5.6 & 5.0 & 5.8 & 5.8 \\
$1 \mathrm{U}$ & 1.0 & 1.2 & 1.3 & 1.2 \\
\hline \hline
\end{tabular}

The size experiments with the adjusted critical values show that it is useful to correct for the effect of the mean shift. When it is known or likely that the time series contains a mean shift the test gains good size properties and appropriate power results. This is very helpful to know when you consider the additional size distortion if the mean shift is neglected. 
Table 4: Power Experiment for one break at the 5\% level

\begin{tabular}{r|lr|lr|lr}
\hline \hline $\mathrm{d}$ & $0.8 \rightarrow 0.4$ & $0.4 \rightarrow 0.8$ & $0.6 \rightarrow 0.0$ & $0.0 \rightarrow 0.6$ & $0.6 \rightarrow 0.4$ & $0.4 \rightarrow 0.6$ \\
\hline & 83.7 & 96.2 & 96.0 & 77.0 & 58.5 & 54.9 \\
\hline \hline
\end{tabular}

The mean shift model can be extended to a more general mean shift model allowing for a smooth transition. It can be driven by a logistic transition function which is modeling a smooth mean shift between the regimes. All results for the abrupt mean shift stay valid. It is just a special case of the general mean shift model. The model can also be extended to more than one break.

\section{Conclusion}

In this paper we show that the Leybourne et al. (2007) test on a break in persistence becomes biased when the data generating process has a shift in the mean function. The test is therefore not robust against mean shifts. The size of the test is most likely even higher than the chosen significance level. Therefore, the null of no change in persistence is falsely rejected by the test due to mean shifts. Mean shifts do effect the test decision even more when they occur at the extreme ends of the sampling period.

As the test is distorted when a mean shift occurs, it is useful to correct for this effect when it is known or likely to have mean shifts in the data. We give adjusted critical values for the case of one mean shift and provide response curves for them. It is shown that the test has good size and reasonable power properties. 


\section{References}

Banerjee, A., Lumsdaine, R. and Stock, J. (1992): "Recursive and sequential tests of the unit root and trend break hypothesis: theory and international evidence." Journal of Business and Economics Statistics 10, 271 - 288.

Belaire-Franch (2005): "A proof of the power of Kim's test against stationary processes with structural breaks.” Econometric Theory 21, 1172 - 1176.

Kim, J. (2000): "Detection of change in persistence of a linear time series." Journal of Econometrics 95, 97 - 116.

Leybourne, S., Taylor, R. and Kim, T. (2007): "CUSUM of squares-based tests for a change in persistence." Journal of Time Series Analysis 28, 408 - 433.

R Development Core Team (2008): 'R: A language and environment for statistical computing." Available at www.r-project.org.

Sibbertsen, P. (2004): "Long memory versus structural breaks: An overview." Statistical Papers 45, $465-515$.

Sibbertsen, P. and Kruse, R. (2009): "Testing for a break in persistence under long-range dependencies." Journal of Time Series Analysis 30, 263 - 285. 


\section{Appendix}

\section{Proof}

\section{Proof of Theorem 1:}

1. Let us first assume that $0.5<d<1.5$. Let us furthermore assume that $\tau \leq \lambda$. The case $\tau \geq \lambda$ is analogous with an interchange of the forward and reverse statistics.

The main advantage of our simple break point model is that we only have to consider the case of a de-meaning of the time series. Due to the fact that a level shift occurs we consider the case of de-meaning instead of de-trending which would be appropriate in the case of a broken trend. For the residuals of (1) we have before the persistence break

$$
\hat{e}_{j}=\varepsilon_{j}-[\tau T]^{-1} \sum_{t=1}^{[\tau T]} \varepsilon_{t}
$$

respectively afterwards

$$
\hat{e}_{j}=\varepsilon_{j}-[(1-\tau) T]^{-1} \sum_{t=[\tau T]+1}^{T} \varepsilon_{t}-[(1-\tau) T]^{-1} \delta \sum_{t=[\tau T]+1}^{T} D_{t}+\delta D_{j} .
$$

Assume $\tau \leq b \leq \lambda$ and $t=[b T]$. For a fixed $\tau$ the mean shift is behind the assumed persistence shift and thus the forward statistics remains unchanged:

$$
K^{f}(\tau)=[\tau T]^{-2 d_{0}} \sum_{t=1}^{[\tau T]} \hat{v}_{t, \tau}^{2} \rightarrow L_{d}^{f}(\tau)
$$

Have in mind that the test always works under the alternative and therefore the existence of a persistence shift is assumed.

For the reversed statistic $\tilde{v}_{t, \tau}$ we obtain:

$$
\begin{aligned}
\tilde{v}_{[b T], \tau}= & \tilde{v}_{[\lambda T], \tau}+\tilde{v}_{[\lambda T]+1, \tau} \\
= & \sum_{j=1}^{T-[\lambda T]} \varepsilon_{j}-\sum_{j=1}^{T-[\lambda T]} \bar{\varepsilon}-\delta \sum_{j=1}^{T-[\lambda T]} \bar{D} \\
& +\sum_{j=T-([\lambda T]+1)}^{T-[b T]} \varepsilon_{j}-\sum_{j=T-([\lambda T]+1)}^{T-[b T]} \bar{\varepsilon}-\delta \sum_{j=T-([\lambda T]+1)}^{T-[b T]} \bar{D}+\sum_{j=T-([\lambda T]+1)}^{T-[b T]} \delta D_{j}
\end{aligned}
$$


with $\bar{\varepsilon}$ and $\bar{D}$ being the mean of $\varepsilon$ and $D$ over the respective time interval.

If $\lambda \leq \tau$ the reversed statistic remains unchanged and we have for the forward statistic:

$$
\begin{aligned}
\hat{v}_{[b T], \tau}= & \hat{v}_{[\lambda T], \tau}+\hat{v}_{[\lambda T]+1, \tau} \\
= & \sum_{j=1}^{[\lambda T]} \varepsilon_{j}-\sum_{j=1}^{[\lambda T]} \bar{\varepsilon}-\delta \sum_{j=1}^{[\lambda T]} \bar{D} \\
& +\sum_{j=[\lambda T]+1}^{[b T]} \varepsilon_{j}-\sum_{j=[\lambda T]+1}^{[b T]} \bar{\varepsilon}-\delta \sum_{j=[\lambda T]+1}^{[b T]} \bar{D}+\sum_{j=[\lambda T]+1}^{[b T]} \delta D_{j} .
\end{aligned}
$$

The statistic is minimized over all $\tau \in \Lambda$ up to $\lambda$ in the first situation and afterwards in the second. This means that up to $\tau=\lambda$ the forward statistic remains unchanged and afterwards the mean shift will effect the residuals by reason that the de-meaning has to consider the mean shift. Thus, for $\tau>\lambda$ the square of the forward statistic increases and therefore the minimum is in the interval $\tau \leq \lambda$ and it is greater or equal the minimum which is obtained without a mean shift.

We have a similar argument for the reversed statistic. For $\tau>\lambda$ it remains unchanged. The changing mean does not affect the recursive de-meaning and thus the residuals remain unchanged. For $\tau<\lambda$ the reversed statistic increases and the minimum is thus in the interval $\tau \geq \lambda$. This proves the first part of the theorem.

2. Let us finally consider the case where $0 \leq d<0.5$. Because of the arguments used before, the minimum of the forward statistic is located earlier than $\lambda$ and that of the backward statistic later than $\lambda$. Therefore, we are in a similar situation as in Sibbertsen and Kruse (2009), Theorem 4, and can therefore adopt the same arguments as in their proof. $\diamond$ 